\title{
STELLAR POSITIONS FROM CCD IMAGES
}

\author{
A. R. Upgren, \\ Van Vleck Observatory, Wesleyan University \\ C. Abad and J. Stock \\ CIDA, Merida
}

The CIDA visual refractor of $65-\mathrm{cm}$ aperture and focal length $10.5 \mathrm{~m}$, has been used extensively for position determinations on photographic plates. The combination of Kodak D plates and a yellow filter permit an almost perfect adaptation to the focal curve of the telescope. It appeared of interest to test whether the telescope could be used for astrometric purposes with a CCD detector. As is well known, the spectral sensitivity of these detectors extends well into the infrared where the images formed by the telescope optics will be far out of focus. The blue spectral region where this would also be the case can easily be cut off by a yellow filter. There are no filters which would produce a similarly sharp cut-off towards the red region. On the other hand, given the small field covered by a CCD, the displacement of the red out-of-focus image with respect to the center of the visual image might be negligible. Recently obtained accurate positions in the area of the Perseus Double Cluster made this field suitable for the test of this possibility.

A number of methods has been developed to determine the center of a stellar image on a CCD exposure. All require that an image extends over a number of pixels. Basically two different procedures can be used, namely a) to adapt a given profile such as a Gaussian distribution to the image, or b) to construct an image model in tabular form and adapt it to the actual images. We have followed both procedures.

Usually a two-dimensional Gaussian image profile is considered the most adequate mathematical representation of true stellar images. This leads to six unknowns of which one describes the central intensity, one the orientation of the major axis of the image, two describe the length of the major and the minor axes of the image, and two more the coordinates of its center.

A new dimension can be added to the formation of artificial image profiles by making use of different functions. We found that the use of the hyperbolic tangent function offers a new flexibility in the profile adaptation. We shall demonstrate its possibilities first in the case of a single dimension. An expression of the form

$$
\mathrm{d}=\mathrm{a} \exp \left[-\left(\mathrm{x}-\mathrm{x}_{\mathrm{o}}\right)^{2} / \mathrm{b}^{2}\right]
$$

represents a Gaussian profile, where $d$ is the intensity, $x$ is a coordinate, $x_{o}$ is the coordinate of the center of the profile with maximum intensity, $a$, and a constant, $b$, which determines the 
width of the profile. It is obvious that no matter which values are used for the different parameters, the resulting profile will always have the shape of a Gaussian distribution. From the expression

$$
\mathrm{d}=\{\tanh [\mathrm{p}(\mathrm{x} / \mathrm{c}+1)-\tanh [\mathrm{p}(\mathrm{x} / \mathrm{c}-1)]\} /(2 \tanh \mathrm{p})
$$

a much larger variety of profiles can be derived. They can in fact simulate a Gaussian at one extreme, and flat-topped profiles with steep edges at the other. The parameter $p$ determines the slope of the edges of the image, while the parameter $\mathrm{c}$ determines its width. This flexibility is obtained by the addition of only one free parameter. In fact, if one would be satisfied with a given form of the profiles, i.e. with one constant value of $p$, the number of free parameters would be identical to those of Gaussian profiles. Naturally, the profile given in equation (2) can readily be extended to two-dimensional elongated images in the same way as is done for Gaussian profiles. Here we should mention that the arctangent function can equally well be used for this purpose. Again, if the two-slope parameters are kept constant, the number of free parameters is reduced to six, as is the case for the Gaussian model.

In the case of CCD images, one may assume that all image profiles are based on one and the same underlying master profile, unlike photographic images. The first step for any CCD exposure should be to find the master profile. For that purpose one may use the image of a bright star, provided one is certain that it is not a double star or an object surrounded by luminous nebulosity. It is safer, though, to co-add the images of several stars, taking care that they are well centered, one on top of the other, before adding. The co-adding process requires some interpolation since the image centers will hardly ever fall right in the middle of a pixel. This is best done by the creation of intermediate pixels which we found to be beneficial for the centering programs. Some smoothing can be done at this point if that is considered to be practical.

The next step is to determine whether an artificial profile created by the mathematical procedure explained above can be satisfactorily adapted to the master profile. We found no appreciable difference in the wings of the profiles for stars of different colors, as might be expected on the basis of the focal curve of the telescope. However, we must admit that the range of colors of the stars investigated is rather small.

The centering is done by an iterative process, starting out with approximate values for all of the parameters involved including the coordinates, and calculating by least squares their most probable corrections. These are applied, and the process is repeated until the corrections become negligible. Naturally, the process makes use of the partial derivatives of the function in use with respect to all of its parameters. This necessity is in fact the reason why it is practical to have a mathematical expression which describes the image profile.

The master profile may not always be fitted by any of the expressions mentioned above. The centering can then be obtained by a strictly numerical process. One must first find a scale factor which will scale the master profile down to the amplitude of the image that is being analyzed. Then by shifting the master profile back and forth in $\mathrm{x}$ and in $\mathrm{y}$ one can find the position of the latter with respect to the real image for which the rms difference becomes a minimum. No significant differences in the positions for bright objects appear upon use of either method. For the faintest stars we found non-systematic differences up to a few hundredths of a pixel. One pixel corresponds to approximately 0.5 arcseconds. 